\title{
Histopathological Changes of Rainbow Trout After \\ Experimental Infection with Lactococcus Garvieae
}

\begin{abstract}
The purpose of this study is to determine both clinical and histopathological changes in experimentally infected rainbow trout with Lactococcus garvieae. Fourty healthy fish samples (50-60 $\mathrm{g}$ in weight) were purchased from a commercial farm near Antalya, Turkey and transferred to the laboratory. Each group of fish were challenged a cell density of $6 \times 10^{5} \mathrm{cfu} / \mathrm{ml}$ of L. garvieae isolate. The control group was given with the appropriate dilution of the sterile PBS. Fish showed darkening of skin color, lesion on the upper jaw and prolapsus. Internally, they had pale liver and kidney, enlarged spleen and hemorrhagies on the muscle. The control group did not show clinical signs. Histopathologically, the kidney and liver showed haemosiderin deposits. Lamellar fusion and hyperplasia of primary lamellae in the gills, depletions of red and white pulpas in the spleen and necrosis of the gastro-intestinal tract were detected.
\end{abstract}

Key Words: Rainbow trout, Lactococcus garvieae, Experimental study

${ }^{1}$ Faculty of Fisheries, Aquaculture Department, Akdeniz University

${ }^{2}$ Faculty of Fisheries, Aquaculture Department, Istanbul University

Correspondence

Jale KORUN

jalekorun@akdeniz.edu.tr

Article Info Received: 25-08-2017 Accepted: 27-10-2017 


\section{Introduction}

Rainbow trout (Oncorhynchus mykiss) is one of the economically important fish species around the world. Production of the species is affected by a variety of factors. These factors include infectious diseases. The infectious diseases are caused by viruses, fungi, bacteria and protozoan parasites. Lactococcosis is a limiting problem for rainbow trout culture in many countries including Turkey and Iran (Sharifiyazdi, et al., 2010; Didinen, et al., 2014). Lactococcus garvieae is the causative agent of Lactococcosis. This pathogen was previously referred as Streptococcus garvieae and then; it was proposed as Enterococcus seriolicida (Sharifiyazdi, et al., 2010). L. garvieae is a non-motile, non-sporulating, facultatively anaerobic, catalase and cytochrome oxidase negative and Gram-positive coccus. It can be isolated from a wide range of animal species including cow, water buffalo, cat, dog and fish. It was also reported in human infections (Vendrell, et al., 2006). Although the evolution of Lactococcosis depends on environmental conditions such as water temperature and water microbiological quality, the disease is described as hemorrhagic septicemia (Vendrell, et al., 2006). The mostly observed signs of the natural disease outbreaks in cultured rainbow trout are uni or bilateral exophthalmia, with periocular hemorrhages, loss of eye or eyes in some cases. Internal clinical signs of the disease include hemorrhagic fluid in the peritoneal cavity, pale liver, enlarged spleen and hemorrhages in the liver and muscle (Pereira, et al., 2004; Savvidis, et al., 2007); however, rainbow trout $(80 \pm 10 \mathrm{~g})$ experimentally infected with L. garvieae via immersion route showed exophthalmia, paleness of the gills, hemorrhages in the liver, swim bladder peritoneum, muscle and bloody content in the intestine (Avci, et al., 2014). Many infectious agents produce typical tissue responses. These responses may change as the infection progresses from acute to chronic forms. The observed histological changes in different organs such as kidney, brain and liver tissue sections may give enough information to describe the effect of a particular infective agent (Gupta, et al., 2009; Alsaid, et al., 2013). Histopathological examinations of rainbow trout which was infected with L. garvieae in culture conditions showed significantly lesions in the liver, kidney, spleen, gills, heart and stomach (Altun, et al., 2005; Didinen, et al., 2014). Avci, et al. (2014) reported that the most important histopathological finding in kidney and spleen was the presence of macrophage in fish tissue samples which were experimentally infected with L. garvieae. There is a little histopathological information for both natural and experimental Lactococcosis infections in Turkey. For this reason, the purpose of this study is to challenge rainbow trout with L. garvieae and observe both clinical and histopathological changes in experimentally infected fish.

\section{Materials and Methods}

Forty healthy rainbow trout (50-60 g in weights) were purchased from a commercial fish farm near Antalya, Turkey and the fish were transferred to the AUFF research laboratory. The fish were separated four group. Each group including control group consisted ten fish and the ten fish were placed into $300 \mathrm{~L}$ fiberglass tank. Water temperature was $18{ }^{\circ} \mathrm{C}$ and it was kept constant during the acclimatization period. All fish were acclimatized to the tanks for 2 weeks before challenge and the fish in each tanks were observed in terms of mortalities and clinical sings for possible disease outbreak. During acclimatization period and after 
challenge, all the fish were fed as $3 \%$ of their fish weights at a daily. The fish were starved for $24 \mathrm{~h}$. before challenge. The procedures were reviewed and approved by the Akdeniz University Local Committee on Animal Research Ethics. Number. 2011.10.01. The L. garvieae strains used in the challenge studies were isolated from a survey study on rainbow trouts which was naturally infected streptococcal species (Korun, et al., 2015). The strains (L1, L2 and L3) were Grampositive, non-motile, cytochrome oxidase and catalase negative. They produced $\alpha$-hemolysis on the blood agar. PLG1 and PLG2 primers (5'CATAACAATGAGAATCGC-3') and PLG-2 (5'GCACCCTCGCGGGTTG-3') were used for the amplification of $16 \mathrm{~S}$ rDNA isolated from the strain (Ravelo, et al., 2003) and we got 1100 bp lenght unique and clear PCR product for the strains. Streptococcus agalactieae (ATCC 12401-20), S. pyogenes (ATCC 19615), Lactococcus garvieae (ATCC 43921) and Gram-negative bacterium, Aeromonas hydrophila (ATCC 19570) were also used as referans for bacterial identification. The amplification products for reference strains were not observed. According to the phenotypical tests and PCR assay results, the isolated strain from moribund rainbow trout was identified as L. garvieae. The challenge procedure was followed that described by Sharifiyazdi, et al. (2010). Briefly, after 2 weeks acclimatization period, the fish samples were challenged by immersion in a strain of L. garvieae grown on BHI agar. Each group of fish except control group were immersed in a bacterial suspension of $6 \times 10^{5}$ colony forming unit/ml of the strain and kept for $30 \mathrm{~min}$ into aquariums. The control group was treated with the appropriate dilution of sterile PBS (Phosphate Buffered Saline). Then, the fish were kept in aerated fiberglass tanks until clinical signs of Lactococcosis and/or fish losses were observed. During this period, the water temperature was maintained at $24^{\circ} \mathrm{C}$. When the clinical signs were evident, moribund fish were subjected to bacterial re-isolation and for histopathological examination, tissue specimens including gills and internal organs from samples were fixed in $10 \%$ buffered formalin solution. The fixed tissues were washed in tap water and dehydrated in the ascending concentrations of ethanol. After dehydration, tissues were cleared in xylene and sectioned at $5 \mu \mathrm{m}$. Then, the sections were stained with hematoxylin and eosin (H+E) (Culling, 1963).

\section{Results and Discussion}

L. garvieae is one of the major Gram-positive coccus pathogen for freshwater salmonid and marine culture species (Khamesipour, et al., 2014). In Turkey, Lactococcosis was firstly reported in cultured rainbow trout (Diler, et al., 2002) and since then, the disease has been appeared regularly in trout farms especially spring and summer seasons (Kav \& Erganis, 2007; Avci, et al., 2010).

Ürkü \& Timur (2014) reported fish which were experimentally injected via intraperitoneal injection of L. garvieae isolates showed loss of orientation, bilateral exophthalmia with periocular hemorrhage, darkening of the skin, enlargement of the spleen, congestion in the liver, intestine and swim bladder. In the present study, three L. garvieae isolates were found to cause same clinical signs on experimentally infected rainbow trout. The all fish started to show lethargy, loss of orientation and swam near to the tank side on the first day of post inoculation (Fig. 1A.). Fish losses from the tank 1 were observed and the fish exhibited darkening of the skin color on the 2 nd day of the trial (Fig. 1B.). Fish from the tank 2 and tank 3 had similar clinical findings of Lactococcosis after 3 days post infection. They had dropsy, exophthalmia, lesions on the upper jaw and anal prolapsus. Internally, they showed pale kidney, 
splenomegaly, pale and hemorrhagic liver (Fig. 2A.). Hemorrhagies on the muscle and air bladder were also observed. Fish from the tank 1 had bilateral exophthalmia and hemorrhagies on the eyes (Fig. 2B.), ascites and anal prolapsus on the 4th day of the trial. Internally, they showed general hemorrhagic septicaemia. The control group did not show clinical findings and / or mortality and histopathological changes. Avci, et al. (2014) informed rainbow trout experimentally infected with L. garvieae via immersion route showed exophthalmia, paleness of the gills, hemorrhagies in the liver, swim bladder peritoneum and muscle, and bloody content in the intestine. This indicate that immersion or injection route of L. garvieae isolates caused same clinical findings and our experimentally infected fish were similar to the findings of Avci, et al. (2014) and Ürkü \& Timur (2014).

After 3 days post infection, the fish inoculated with L. garvieae showed disease signs and mortality and the infected fish were used for re-isolation of the isolates. The isolates produced small, ovoid-cocci and white color colonies after incubation at $24 \pm 2{ }^{\circ} \mathrm{C}$ for 48 hours. The isolates were fermentative, Gram-positive, cytochrome oxidase and catalase negative. Lactococcus garvieae (ATCC 43921) was also included as reference strain in the bacteriological study. The all phenotypic test results which were produced by the isolates are given in Table 1. Positive PCR amplification was obtained with L. garvieae using specific primers (PLG1 and PLG2) for a fragment of the $16 \mathrm{~S}$ rDNA. The amplification of the target gene for L. garvieae permitted identification of the isolates as L. garvieae with 1100 bp (Fig. 3.). According to the results of bacteriological and molecular studies, L. garvieae was re-isolated from the experimentally diseased fish samples.
In the present study, all experimentally infected fish challenged with L. garvieae had changes in the gill, liver, kidney, spleen and gastro-intestinal tract. The gill showed lamellar fusion, hyperplasia of primary lamellae and epithelial necrosis (Fig. 4.). The liver showed hemorrhagy and increasing of brown haemosiderin deposits (Fig. 5.). Histopathological examination of kidney tissue exhibited heavy haemosiderin deposits and liquefactive necrosis of kidney tubules (Fig. 6.). The spleen tissue showed depletions of red and white pulpas (Fig. 7.). Necrosis of the gastro-intestinal tract was also detected. Ürkü \& Timur (2014) found multifocal necrosis and hemorrhages in the liver, kidney and spleen, and talengiectiasis in the gills. Avci, et al. (2013) informed oedema and swelling in the secondary lamellar epithelium in the gills, focal or multifocal coagulation necrosis in the spleen. In this study, our histopathological findings such as hemorrhages in the liver and necrosis of kidney tubules were similar to the findings of Ürkü \& Timur (2014). Talengiectiasis and multifocal coagulation necrosis (Avci, et al. (2014) were not observed in this study.

Results obtained in this study indicated that experimentally infected fish showed same clinical signs in other experimental studies; however, histopathological findings of tissue samples in this study were different findings which were reported in the other studies. It was understood that L. garvieae caused different histopathological changes in the tissue samples.

\section{Acknowledgments}

This work was supported by Akdeniz University Scientific Research Projects (BAP) Unit under Project No: 2011.01.0111.001. 


\section{Table 1. Phenotypic properties of reference strain (L. garvieae, ATCC 43921) and isolates ( $\mathrm{n}=8$ )}

\begin{tabular}{|c|c|c|}
\hline Character & Isolates $(\mathrm{n}=8)$ & $\begin{array}{l}\text { L. garvieae, reference strain } \\
\text { (ATCC 43921) }\end{array}$ \\
\hline Cell morphology & ovoid-cocci & ovoid-cocci \\
\hline Motility & - & - \\
\hline Colony colour & $\mathrm{w}^{\mathrm{a}}$ & $\mathrm{W}$ \\
\hline Gram-staining & + & + \\
\hline $\mathrm{O} / \mathrm{F}$ (glucose) & $\mathrm{F}^{\mathrm{b}}$ & $\mathrm{F}$ \\
\hline \multicolumn{3}{|l|}{ Production of: } \\
\hline C. oxidase & - & - \\
\hline Catalase & - & - \\
\hline Indole & - & - \\
\hline $\mathrm{H}_{2} \mathrm{~S}$ & - & - \\
\hline $\mathrm{ADH}$ & + & + \\
\hline LDC & - & - \\
\hline ODC & - & - \\
\hline Nitrate reduction & - & - \\
\hline Voges-Proskauer & + & + \\
\hline Metil red & + & + \\
\hline \multicolumn{3}{|l|}{ Degredation of: } \\
\hline Gelatin & - & + \\
\hline Starch & - & - \\
\hline Blood (hemolysis) & $\alpha$ & $\alpha$ \\
\hline Citrate utilization & - & - \\
\hline \multicolumn{3}{|l|}{ Growth at: } \\
\hline $4^{\circ} \mathrm{C}$ & - & - \\
\hline $37^{\circ} \mathrm{C}$ & + & + \\
\hline $45^{\circ} \mathrm{C}$ & + & + \\
\hline \multicolumn{3}{|l|}{ Growth in: } \\
\hline $0 \%-2 \% \mathrm{NaCl}$ & + & + \\
\hline $4 \%-6 \% \mathrm{NaCl}$ & + & + \\
\hline $6.5 \% \mathrm{NaCl}$ & + & + \\
\hline ONPGc & - & - \\
\hline \multicolumn{3}{|c|}{ Production acid from: } \\
\hline Arabinose & - & - \\
\hline Glucose & + & + \\
\hline Inositol & + & + \\
\hline Fructose & + & + \\
\hline
\end{tabular}


Galactose

Mannitol

Mannose

Sorbitol

Sucrose

Xylose

$\begin{array}{lr}+ & + \\ + & + \\ + & + \\ + & + \\ + & - \\ + & +\end{array}$

+: positive, -: negative, a: white, b: fermentative

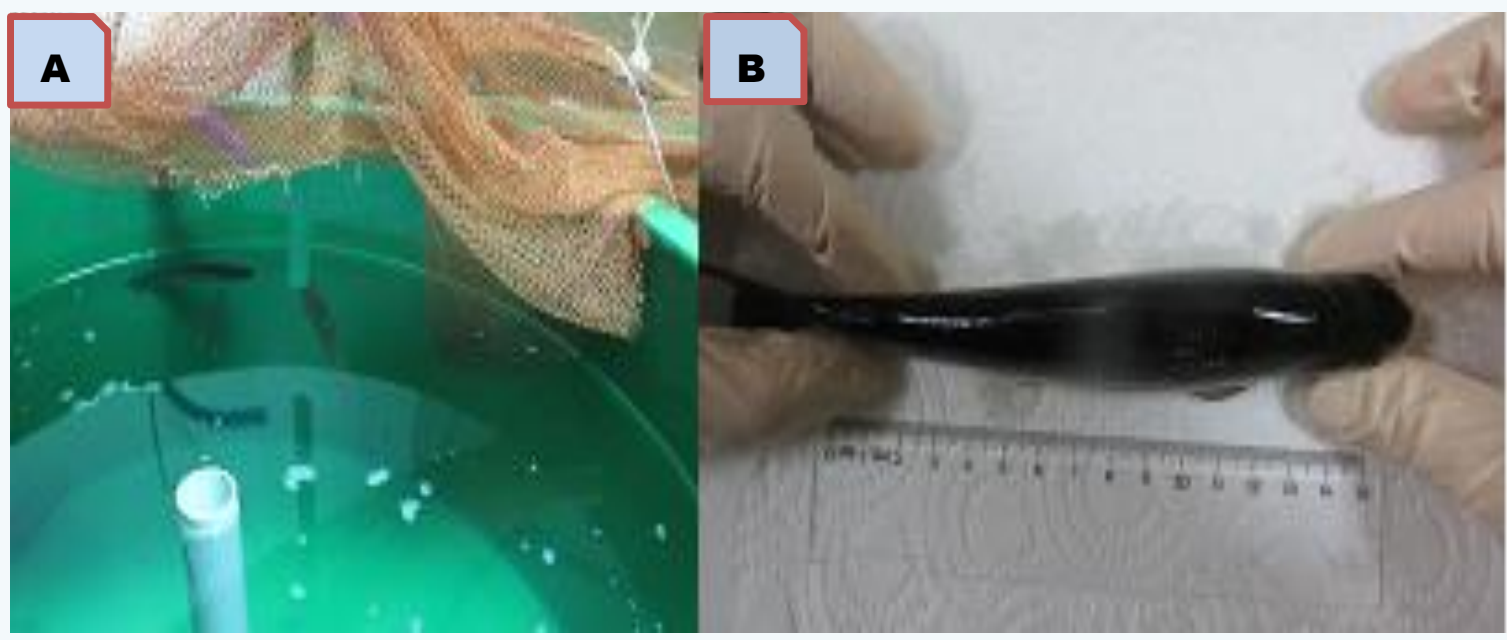

Figure 1A: Infected fish showed loss of orientation on the $1^{\text {st }}$ day of post oculation Figure 1B: Darkening of the skin color on the $2^{\text {nd }}$ day of the trial

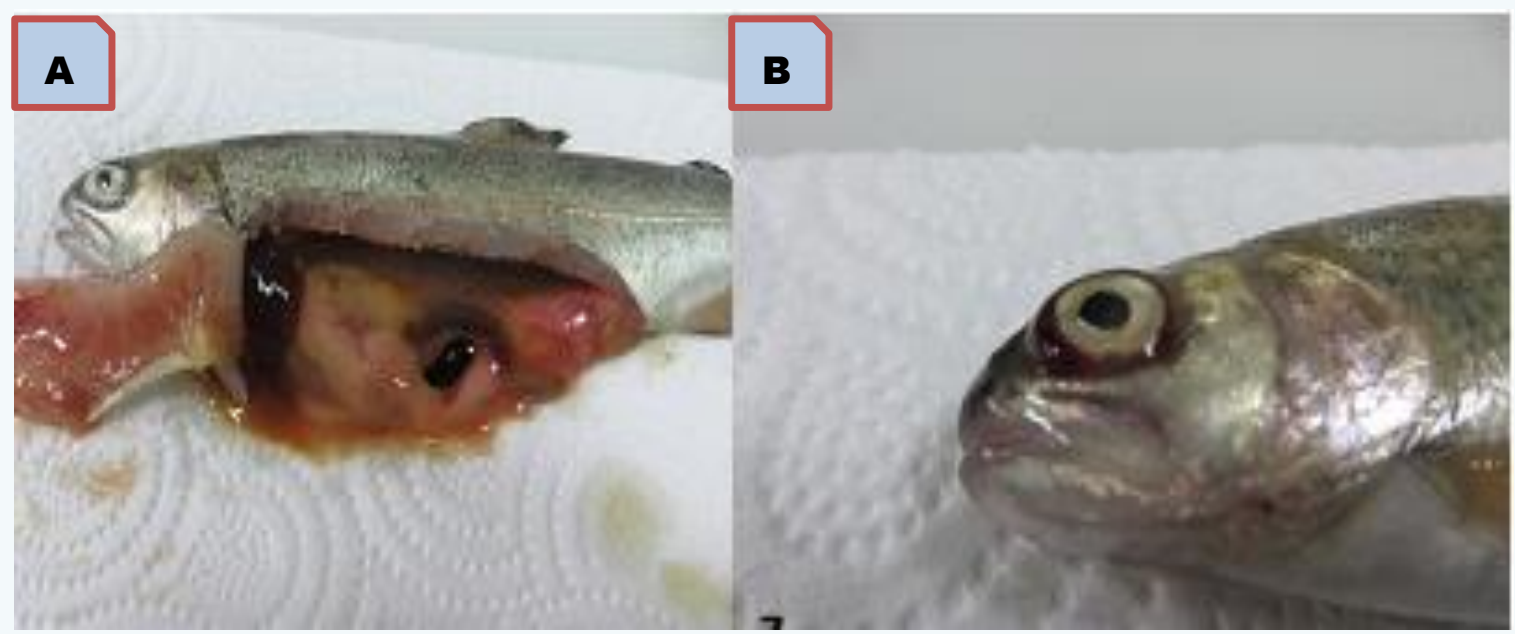

Figure 2A: Splenomegaly and hemorrhagic liver on the 3rd day of the trial

Figure 2B: Bilateral exophthalmia and hemorrhagies on the eyes on the 4th day of trial 


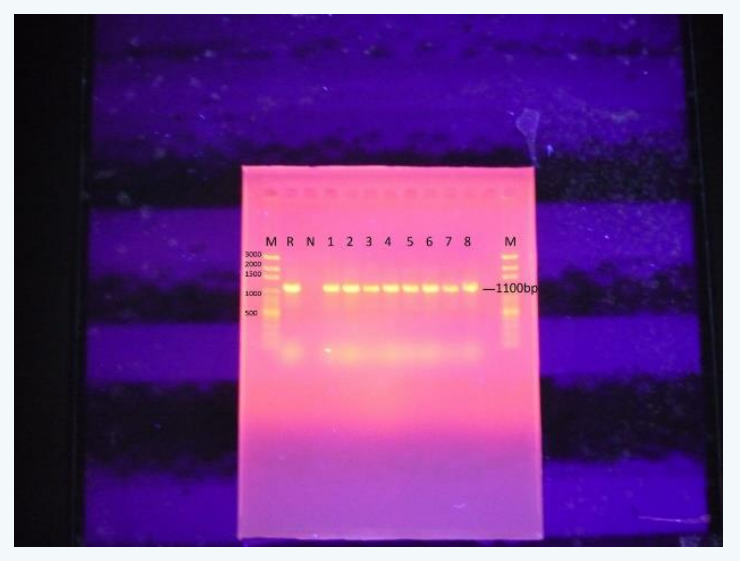

Figure 3: Amplification products of the isolates from the experimentally infected rainbow trout in the study. M: Marker, Solip BioDyne 100 bp DNA ladder; R: positive control, L. garvieae, (ATCC 43921); N: negative control, double distilled water

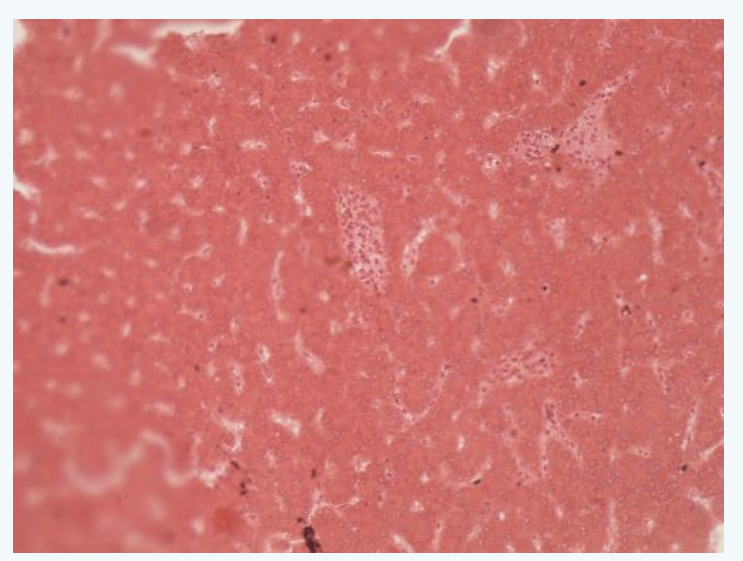

Figure 5: Hemorrhagies (arrow) in the liver (H+E x 20)

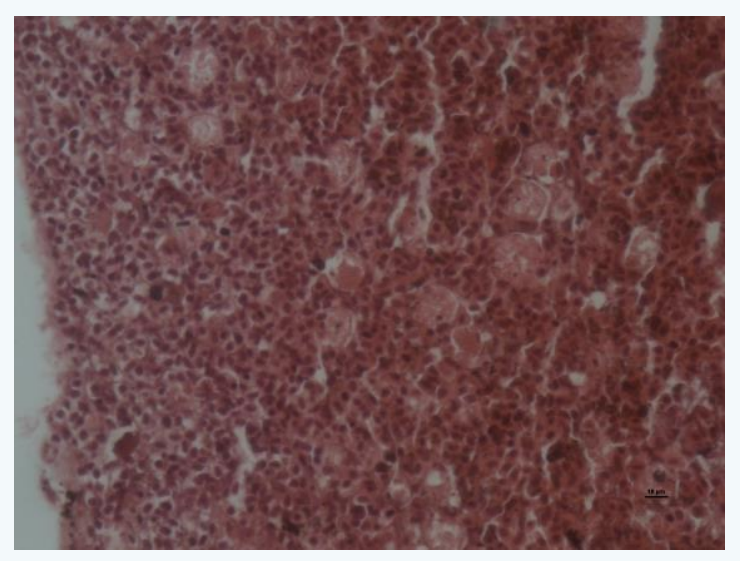

Figure 7. Depletions of red and white pulpas in the spleen $(\mathrm{H}+\mathrm{E} \times \mathrm{40})$

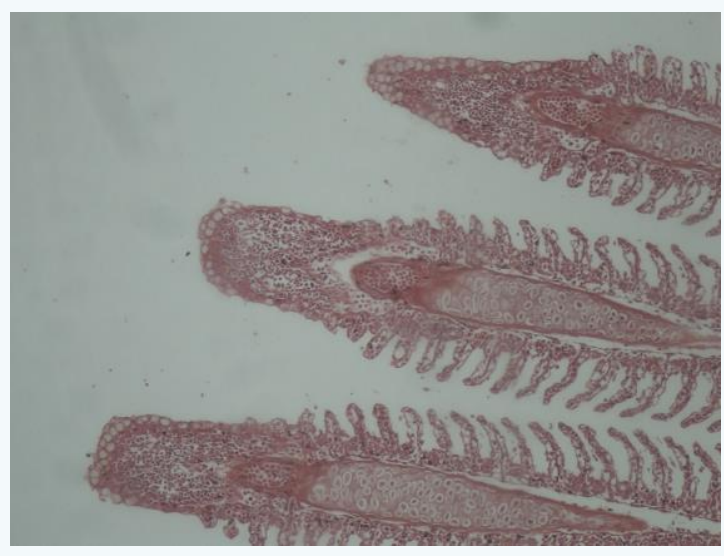

Figure 4: Gill of rainbow trout infected with $L$. garvieae. The gill showed lamellar fusion, hyperplasia (arrow) and epithelial necrosis (H+E x 20)

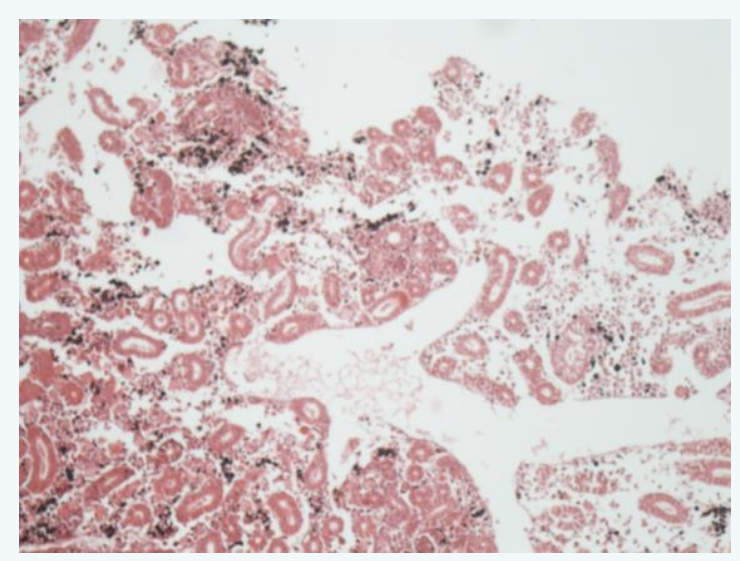

Figure 6: Massive haemosiderin deposits (arrow) and liquefactive necrosis in kidney (H+E x 10) 


\section{References}

Alsaid, M., Daud, H. H. M., Mustapha, N. M., Bejo, S. K., Abdelhadi, Y. M., Abuselina, A. F., \& Hmdan, R. H. (2013). Pathological findings of experimental Streptococcus agalactiae infection in red hybrid tilapia (Oreochromis sp.). International Conference on Chemical, Agricultural and Medical Sciences (CAMS-2013), Kuala Lumpur (Malaysia), 70-73.

Altun, S., Diler, A., Diler, Ö., Başak, K., \& Işıklı, B. I. (2005). Histopathology of streptococcosis in rainbow trout (Oncorhynchus mykiss, Walbaum). Bulletin of the European Association of Fish Pathologists, 25(3), 131-135.

Avci, H., Aydoğan, A., Tanrıkul, T. T., \& Birincioğlu, S. S. (2010). Pathological and microbiological investigations in rainbow trout (Oncorhynchus mykiss Walbaum, 1792) naturally infected with Lactococcus garvieae. Kafkas Üniversitesi Veteriner Fakültesi Dergisi, 16, 313-318.

Avci, H., Birincioğlu, S. S., Tanrıkul, T.T., Epikmen, T. T., Metin, N., \& Avsever, M. L. (2014). Experimental Lactococcus garvieae infection in rainbow trout, Oncorhynchus mykiss, Walbaum 1792: a comparative histopathological and immunohistochemical study. Journal of Fish Diseases, 37, 481-495.

Culling C. F. A. (1963). Handbook of histopathological techniques. England: Butter Worth Publishing.

Didinen, B. I., Yardımcı, B., Onuk, E. E., Metin, S., \& Yıldırım, P. (2014). Naturally Lactococcus garvieae infection in rainbow trout (Oncorhynchus mykiss Walbaum, 1792) new histopathological observations, phenotypic and molecular identification. Revue de Medecine Veterinaire, 165(1-2), 12-19.

Diler, O., Altun, S., Adiloğlu, A., Kubilay, A., \& Işıklı, B. (2002). First occurrence of streptococcosis affecting farmed rainbow trout (Oncorhynchus mykiss) in Turkey. Bulletin of the European Association of Fish Pathologists, 22, 21-26.

Gupta, E., Bhalla, P., Khurana, N., \& Singh, T. (2009). Histopathology for the diagnosis of infectious diseases. Indian Journal of Medical Microbiology, 27(2), 100-106.

Kav, K., \& Erganiş, O. (2007). Konya Bölgesinde bulunan gökkuşağı alabalığı (Oncorhynchus mykiss) çiftliklerinden Lactococcus garvieae izolasyonu, identifikasyonu ve fenotipik özelliklerinin belirlenmesi. Veteriner Bilimleri Dergisi, 23, 7-17.

Khamesipour, F., Shahrani, M., Raki, A., \& Doosti, A. (2014). Detection of Lactococcus garvieae and Vibrio cholera in some aquatic fishes of Persian Gulf using polymerase chain reaction. European Journal of Experimental Biology, 4(3), 44-49.

Korun, J., Timur, G., Yardımcı, R. E., \& Balci, B. A. (2015). Determination of infections on the cultured rainbow trout (Oncorhynchus mykiss, W) caused by some streptococcal species. Akdeniz University Scientific Research Project Coorodination Section, Project Number: 2011.01.0111.001, 50 p., Antalya.

Pereira, F., Ravelo, C., Toranzo, A. E., \& Romalde, J. L. (2004). Lactococcus garvieae, an emerging pathogen for the Portuguese trout culture. Bulletin of the European Association of Fish Pathologists, 24(6), 274-279.

Ravelo, C., Magariños, B., López-Romalde, S., \& Toranzo, A. E. (2003). Molecular fingerprintings of fish-pathogenic Lactococcus garvieae strains by random amplified polymorphic DNA analysis. Journal of Clinical Microbiology, 41, 751-756.

Savvidis, G. K., Anatoliotis, C., Kanaki, Z., \& Vafeas, G. (2007). Epizootic outbreaks of Lactococcosis disease in rainbow trout, Oncorhynchus mykiss (Walbaum), culture in Greece. Bulletin of the European Association of Fish Pathologists, 27(6), 223-228.

Sharifiyazdi, H., Akhlaghi, M., Tabatabaei, M., \& Mostafavi, S. M. (2010). Isolation and characterization of Lactococcus garvieae from diseased rainbow trout (Oncorhynchus mykiss, Walbaum) cultured in Iran. Iranian Journal of Veterinary Research, Shiraz University, 11(33), 342-350.

Ürkü, Ç., \& Timur, G. (2014). A comparative study of detection methods for Lactococcus garvieae in experimentally infected rainbow trout (Oncorhynchus mykiss, W.). The Israeli Journal of Aquaculture-Bamidgeh, IJA-66.2014.1025, 10 pages.

Vendrell, D., Balcázar, J. L., Ruiz-Zarzuela, I., Blas, de I., Gironés, O., \& Múzquiz, J. L. (2006). Lactococcus 
garvieae in fish: A review. Comparative Immunology,

Microbiology\&Infectious Diseases, 29, 177-198. 